\title{
Contributing ocular comorbidity to end-of-life visual acuity in medically treated glaucoma patients, ocular hypertension and glaucoma suspect patients
}

\author{
Palwasha Mokhles $\mathbb{D}^{1} \cdot$ Luuk van Gorcom ${ }^{1} \cdot$ Jan S. A. G. Schouten $\mathbb{D}^{1,2} \cdot$ Tos T. J. M. Berendschot ${ }^{1}$ • \\ Henny J. M. Beckers ${ }^{1} \cdot$ Carroll A. B. Webers ${ }^{1}$
}

Received: 21 November 2019 / Revised: 16 May 2020 / Accepted: 19 May 2020 / Published online: 3 June 2020

(c) The Author(s), under exclusive licence to The Royal College of Ophthalmologists 2020

\begin{abstract}
Aim To assess the visual acuity at the end of life in glaucoma suspect patients, ocular hypertension, and patients treated for glaucoma and to find factors contributing to a reduced visual acuity in this cohort of deceased patients.

Methods In a cohort of 3883 medically treated glaucoma patients, glaucoma suspect, or patients with ocular hypertension assembled in 2001-2004, 1639 were deceased. Patient data were collected from electronic and paper patient files. The files of 1378 patients were studied and the last measured visual acuity and ocular comorbidities influencing the visual acuity were extracted.

Results Our results show that only $37.2 \%$ of patients had no visual impairment in either eye, $30.5 \%$ was visually impaired or blind in both eyes and $4.1 \%$ was blind in both eyes, all based on VA. The most common contributing factors for severe visual impairment or blindness (prevalence $\geq 1 \%$ ) were: glaucoma, retinal vein occlusion, dry and exudative age-related macular degeneration, past retinal detachment, amblyopia, diabetic retinopathy, anterior ischemic optic neuropathy, trauma, decompensated cornea, past keratitis, enucleation, corneal transplantation, and macular hole.

Conclusions Despite the current advanced treatment modalities for glaucoma, 30.5\% of patients had a VA $<0.5$ in both eyes and $4.1 \%$ was blind in both eyes. However, this disability cannot be confidently attributed only to glaucoma. Besides glaucoma, most common contributing factors were among others retinal and macular diseases. Patient management in glaucoma should be based on more than lowering the intraocular pressure to prevent blindness at the end of life.
\end{abstract}

\section{Introduction}

Glaucoma is the major cause of irreversible blindness worldwide [1-4]. The global prevalence of glaucoma has increased due to the aging population. It is therefore expected that the number of patients with visual impairment (VI) or blindness due to glaucoma, but also other eye diseases, will likewise rise in the coming years [5-7]. Glaucoma treatment is solely focused on reducing the intraocular

Supplementary information The online version of this article (https:// doi.org/10.1038/s41433-020-0991-0) contains supplementary material, which is available to authorized users.

\footnotetext{
Palwasha Mokhles

palwasha.mokhles@mumc.nl

1 University Eye Clinic Maastricht, Maastricht, The Netherlands

2 Canisius Wilhelmina Ziekenhuis, Nijmegen, The Netherlands
}

pressure in order to reduce the progression of visual field (VF) loss and to prevent glaucoma blindness at the end of life [2-4]. However, to prevent a blind death in glaucoma patients, the focus of treating glaucoma could need a paradigm shift, if the treatment of glaucoma is already very successful and the cause of a blind death is related to other eye diseases. Any patient with glaucoma is at risk of developing another eye disease such as cataract and agerelated macular degeneration (AMD), and this risk increases as the life expectancy increases $[8,9]$. Moreover, some eye diseases are more common in glaucoma, e.g., retinal vein occlusion (RVO) of which most commonly central retinal vein occlusion (CRVO) [10-12]. These diseases have a major impact on visual acuity (VA) and the risk of a blind death.

We aimed to study VA at the end of life in glaucoma and its impact on VI and blindness in these patients. Contributing factors to a compromised VA were determined as well. 


\section{Methods}

We performed a retrospective follow-up cohort study by investigating the medical records of deceased glaucoma, glaucoma suspect, or ocular hypertension patients from nine randomly selected hospitals in the Netherlands (academic, teaching, and non-teaching), which were included in the DUtch Research project on treatment outcome IN Glaucoma patients (DURING) study [13]. Patients were included in the original study between 2001 and 2004, the baseline response rate in this study was $79 \%$. Patients were eligible if they received medical treatment for ocular hypertension, glaucoma suspect, or glaucoma. At inclusion patients gave informed consent to access and use their patient records up to 20 years after inclusion. Ethics Committee approval was obtained from the Institutional Review Board from Maastricht University Medical Centre (MUMC+). The current study adhered to the tenets of the Declaration of Helsinki. Of the 3883 patients included in the original DURING study, 1639 were deceased at the last conducted search on Nov 18, 2015. After studying patient records, 1378 patients were included in the analysis for the current study. Due to missing files, 260 patients had to be excluded and one patient withdrew informed consent during the follow-up period.

\section{Data collection}

Medical records were collected between January 2016 and March 2017 and studied between March and June 2017. We extracted the last measured VA and assessed the course of VA during the follow-up period. Furthermore, contributing factors to a lower VA, such as ocular comorbidities and VF loss, were assessed as well. Baseline diagnoses of the type of glaucoma as assessed at inclusion of the patients were collected from the original database of the DURING study and checked for in the patient record if the database was inconclusive.

Contributing diseases were based on reported diagnoses or retrospectively set diagnoses based on the ophthalmologic examination and VF testing from the patient record. In case no other cause than glaucoma was found, and the most recent VF corresponded as such or the foveal sensitivity or central scotoma corresponded with the VA, glaucoma was determined as the most likely contributing factor. In cases were atrophic macular changes were reported, but without the diagnosis of AMD, the diagnosis macular atrophy was recorded. If the evidence was inconclusive, the case was discussed by two of the authors (JSAGS and LVG) and either consensus was reached or 'e cause ignota' (e.c.i.) was noted if no contributory factor could be identified.

We defined VI and blindness according to the classification of the World Health Organization (WHO): mild VI, VA $<0.5$ and $\geq 0.3$; moderate VI, VA $<0.3$ and $\geq 0.1$; severe VI, VA $<$ 0.1 and $\geq 0.05$ and blind, $\mathrm{VA}<0.05$. VA measurements in decimals were converted to Logarithm of Minimal Angle of Resolution (LogMAR) units for statistical analysis. The following LogMAR denotations were used for non-numeric values [14]: counting fingers $(\mathrm{CF})=1.78 \mathrm{LogMAR}$ and hand movement $=2.48$ LogMAR. For light perception $(\mathrm{LP}+)$ and no light perception (LP-) we used 3 LogMAR and 4 LogMAR, respectively.

The data from the medical file were used to assess the presence and influence of contributing factors on the end of life VA. This started with the assessment of the VA at the end of life. Thereafter the VA at the beginning of the file was assessed. Visits after the start of the file were used to determine if there was a change in VA. If there was a change, the reported contributing eye disease was noted. In case no explanation was found, the VF's were used to assess if glaucoma was the contributing factor. The VF defect should then fit the glaucomatous pattern and have extended toward the center of the VF. In case no explaining factor could be found, it was reported as "no contributing factor." In case more than one contributing factor was present during the follow-up, these were ranked according to their presumed impact on the end of life VA. In case the VA was already in a relevant range at study entry, the eye diseases that were reported to have contributed to this low level of VA were noted. Contributing factors were reported individually in the tables if their prevalence was $\geq 1 \%$ as a major contributing factor.

\section{Results}

Table 1 displays characteristics of the deceased population included in the study $(N=1378)$. The table shows the total number $(N)$ and the percentages $(\%)$. Age at death was 83.7 years, the mean time until death was 7.4 years. The results of VI and blindness in the following Tables were based on the VA.

The results of the VA for right and left eye are shown in Table 2. Blindness was present in $14.3 \%$ in the right eye and $15.3 \%$ in the left eye. Blindness was present in both eyes in $4.1 \%$ of the patients and $30.5 \%$ had a VA lower than 0.5 in both eyes.

The prevalence of VI and blindness per glaucoma diagnosis is presented in Table 3. This shows that in patients with primary open angle glaucoma $16.1 \%$ (right eye) and $17.8 \%$ (left eye) die with severe VI or blind. These values are lower in patients with ocular hypertension and do not seem higher in patients with narrow angle glaucoma or normal tension glaucoma. The prevalence of blindness is higher in patients with secondary glaucoma.

Table 4 presents the ocular diseases which contributed to a reduced VA in these patients. It shows the most prevalent relevant ocular disease contributing to the VI or blindness. 
Table 1 Characteristics of the deceased patients included in the study $(N=1378$. Except diagnoses group in which $N=1297)$.

\begin{tabular}{|c|c|c|c|}
\hline Variables & & OD & OS \\
\hline \multicolumn{4}{|l|}{ Gender $(N, \%)$} \\
\hline Female & $671(48.7)$ & - & - \\
\hline Male & $707(51.3)$ & - & - \\
\hline $\begin{array}{l}\text { Age at death in years } \\
\text { (mean, SD) }\end{array}$ & $83.7(8.3)$ & - & - \\
\hline $\begin{array}{l}\text { Age at baseline in years } \\
\text { (mean, SD) }\end{array}$ & $76.3(8.1)$ & - & - \\
\hline $\begin{array}{l}\text { LogMAR VA at the end of } \\
\text { life (mean, SD) }\end{array}$ & - & $0.61(0.93)$ & $0.63(0.92)$ \\
\hline Follow-up time in years & 7.4 & - & - \\
\hline \multicolumn{4}{|l|}{ Type of hospital $(N, \%)$} \\
\hline Regional & $740(53.7)$ & - & - \\
\hline Top-clinical & $323(23.4)$ & - & - \\
\hline Academic & $315(22.9)$ & - & - \\
\hline \multicolumn{4}{|l|}{ Diagnosis at baseline } \\
\hline $\mathrm{POAG}^{\mathrm{a}}$ & - & $874(63.4)$ & $867(62.9)$ \\
\hline POAG suspect & - & $34(2.5)$ & $41(3.0)$ \\
\hline $\begin{array}{l}\text { Conversion } \mathrm{OHT}^{\mathrm{b}} \text { to } \\
\text { POAG (between first and } \\
\text { second hospital visit in } \\
0.5-1 \text { year) }\end{array}$ & - & $19(1.4)$ & $21(1.5)$ \\
\hline OHT & - & $103(7.5)$ & $106(7.7)$ \\
\hline $\mathrm{NTG}^{\mathrm{c}}$ & - & $56(4.1)$ & $59(4.3)$ \\
\hline Primary $N A G^{d}$ & - & $43(3.1)$ & $43(3.1)$ \\
\hline Chronic NAG & - & $22(1.6)$ & $22(1.6)$ \\
\hline Mixed & - & $15(1.1)$ & $11(0.8)$ \\
\hline $\mathrm{PDS}^{\mathrm{e}}$ & - & $7(0.5)$ & $7(0.5)$ \\
\hline$P E X^{f}$ & - & $15(1.1)$ & $17(1.2)$ \\
\hline Secondary & - & $42(3.0)$ & $31(2.2)$ \\
\hline Unclassified & - & $67(4.9)$ & $72(5.2)$ \\
\hline Unknown & - & 81 (4.9) & $81(5.9)$ \\
\hline
\end{tabular}

${ }^{a} P O A G$ primary open angle glaucoma.

${ }^{\mathrm{b}} \mathrm{OHT}$ ocular hypertension.

${ }^{\mathrm{c}} N T G$ normal tension glaucoma.

${ }^{\mathrm{d}} N A G$ narrow angle glaucoma.

${ }^{\mathrm{e}} P D S$ pigment dispersion syndrome.

${ }^{\mathrm{f}} P E X$ pseudo-exfoliation syndrome.
The most common contributing factors for severe VI or blindness (prevalence $\geq 1 \%$ ) were (several forms of) glaucoma, RVO, dry and exudative AMD, past retinal detachment, amblyopia, diabetic retinopathy (DRP), anterior ischemic optic neuropathy, trauma, decompensated cornea, past keratitis, enucleation, corneal transplantation, and macular hole.

In the group with mild to moderate VI the contributing factors with a prevalence of $\geq 1 \%$ were cataract and dry AMD, glaucoma, exudative AMD, posterior capsular opacification, amblyopia, DRP, corneal dystrophy, branch RVO, and (past) keratitis. In a considerable number of cases no contributing factor could be identified. Dry AMD in the more severe group was likely to be geographic atrophy while in the group with milder VI it would be retinal pigment epithelial changes.

In addition, we looked for contributing comorbidities per subgroup as well. However, we limited this to four groups. POAG, POAG suspect + OHT conversion to $\mathrm{POAG}+$ OHT, NAG, NTG, and others. Groups were combined since number per subgroup were small. Even then, the groups are small making it difficult to compare the subgroups for differences in ranking of contributing factors. In comparing POAG with OHT/POAG suspect and conversion, glaucoma as a cause of VI or blindness ranks higher in the PAOG group, as expected. These results are shown in Table 5, which gives the top three most contributing factors for every category of VA per subgroup. These numbers are presented for the right eye only, since the left eye showed the same results.

The prevalence of VI and blindness stratified according to the hospital type are shown in Table 6 (see Supplementary). The prevalence of blindness is higher in the university hospital patients. The prevalence of VI varies in percentage between university and top-clinical hospitals.

\section{Discussion}

A considerable number of patients with glaucoma, glaucoma suspect, or ocular hypertension will die blind or with
Table 2 Prevalence of visual impairment and blindness at the end of life in the right and left eye in a cohort of patients with ocular hypertension, glaucoma suspect or glaucoma, according to the WHO criteria on the base VA.

\begin{tabular}{lllllll}
\hline & OD & & & \\
\cline { 2 - 6 } OS & Normal & Mild VI & Moderate VI & Severe VI & Blind & Total \\
\hline Normal & $513(37.2)$ & $76(5.5)$ & $52(3.8)$ & $14(1.0)$ & $67(4.9)$ & $722(52.4)$ \\
Mild VI & $96(7.0)$ & $73(5.3)$ & $26(1.9)$ & $5(0.4)$ & $20(1.5)$ & $220(16.0)$ \\
Moderate VI & $50(3.6)$ & $37(2.7)$ & $50(3.6)$ & $7(0.5)$ & $42(3.0)$ & $186(13.5)$ \\
Severe VI & $10(0.7)$ & $5(0.4)$ & $9(0.7)$ & $3(0.2)$ & $12(0.9)$ & $39(2.8)$ \\
Blind & $80(5.8)$ & $33(2.4)$ & $35(2.5)$ & $7(0.5)$ & $56(4.1)$ & $211(15.3)$ \\
Total & $749(54.4)$ & $224(16.3)$ & $172(12.5)$ & $36(2.6)$ & $197(14.3)$ & $1378(100)$ \\
\hline
\end{tabular}

VI visual impairment. 
Table 3 Prevalence of visual impairment and blindness at the end of life according to the type of glaucoma in a cohort of patients with ocular hypertension, glaucoma suspect or glaucoma.

\begin{tabular}{|c|c|c|c|c|c|c|c|c|c|c|c|}
\hline & \multicolumn{2}{|c|}{ Normal } & \multicolumn{2}{|c|}{ Mild $\mathrm{VI}^{\mathrm{a}}$} & \multicolumn{2}{|c|}{$\begin{array}{l}\text { Moderate- } \\
\text { VI }\end{array}$} & \multicolumn{2}{|c|}{$\begin{array}{l}\text { Severe- } \\
\text { VI }\end{array}$} & \multicolumn{2}{|c|}{ Blind } & \multirow[b]{2}{*}{ Total } \\
\hline & $n$ & $\%$ & $n$ & $\%$ & $n$ & $\%$ & $n$ & $\%$ & $n$ & $\%$ & \\
\hline \multicolumn{12}{|l|}{ OD } \\
\hline $\mathrm{POAG}^{\mathrm{b}}$ & 477 & 54.6 & 153 & 17.5 & 103 & 11.8 & 21 & 2.4 & 120 & 13.7 & 874 \\
\hline POAG suspect & 21 & 61.8 & 8 & 23.5 & 3 & 8.8 & 0 & 0.0 & 2 & 5.9 & 34 \\
\hline Conversion $\mathrm{OHT}^{\mathrm{c}}$ to $\mathrm{POAG}$ & 13 & 68.4 & 2 & 10.5 & 2 & 10.5 & 0 & 0.0 & 2 & 10.5 & 19 \\
\hline OHT & 71 & 68.9 & 12 & 11.7 & 10 & 9.7 & 4 & 3.9 & 6 & 5.8 & 103 \\
\hline $\mathrm{NTG}^{\mathrm{d}}$ & 29 & 51.8 & 6 & 10.7 & 14 & 25.0 & 0 & 0.0 & 7 & 12.5 & 56 \\
\hline Chronic $\mathrm{NAG}^{\mathrm{e}}$ & 12 & 54.5 & 7 & 31.8 & 1 & 4.5 & 0 & 0.0 & 2 & 9.1 & 22 \\
\hline Primary NAG & 27 & 62.8 & 5 & 11.6 & 7 & 16.3 & 2 & 4.7 & 2 & 4.7 & 43 \\
\hline Mixed & 5 & 33.3 & 4 & 26.7 & 3 & 20.0 & 0 & 0.0 & 3 & 20.0 & 15 \\
\hline $\operatorname{PDS}^{\mathrm{f}}$ & 5 & 71.4 & 1 & 14.3 & 0 & 0.0 & 0 & 0.0 & 1 & 14.3 & 7 \\
\hline $\mathrm{PEX}^{\mathrm{g}}$ & 8 & 53.3 & 3 & 20.0 & 4 & 26.7 & 0 & 0.0 & 0 & 0.0 & 15 \\
\hline Secondary & 8 & 19.0 & 2 & 4.8 & 7 & 16.7 & 4 & 9.5 & 21 & 50.0 & 42 \\
\hline Unclassified & 31 & 46.3 & 9 & 13.4 & 5 & 7.5 & 2 & 3.0 & 20 & 29.9 & 67 \\
\hline Total & 707 & 54.5 & 212 & 16.3 & 159 & 12.3 & 33 & 2.5 & 186 & 14.3 & 1297 \\
\hline \multicolumn{12}{|l|}{ OS } \\
\hline POAG & 448 & 51.7 & 150 & 17.3 & 115 & 13.3 & 25 & 2.9 & 129 & 14.9 & 867 \\
\hline POAG suspect & 22 & 53.7 & 11 & 26.8 & 7 & 17.1 & 0 & 0.0 & 1 & 2.4 & 41 \\
\hline Conversion $\mathrm{OHT}$ to $\mathrm{POAG}$ & 14 & 66.7 & 2 & 9.5 & 1 & 4.8 & 0 & 0.0 & 4 & 19.0 & 21 \\
\hline OHT & 77 & 72.6 & 13 & 12.3 & 7 & 6.6 & 1 & 0.9 & 8 & 7.5 & 106 \\
\hline NTG & 36 & 61.0 & 3 & 5.1 & 9 & 15.3 & 3 & 5.1 & 8 & 13.6 & 59 \\
\hline Chronic NAG & 12 & 54.5 & 4 & 18.2 & 2 & 9.1 & 1 & 4.5 & 3 & 13.6 & 22 \\
\hline Primary NAG & 20 & 46.5 & 12 & 27.9 & 7 & 16.3 & 0 & 0.0 & 4 & 9.3 & 43 \\
\hline Mixed & 6 & 54.5 & 0 & 0.0 & 5 & 45.5 & 0 & 0.0 & 0 & 0.0 & 11 \\
\hline PDS & 3 & 42.9 & 1 & 14.3 & 0 & 0.0 & 0 & 0.0 & 3 & 42.9 & 7 \\
\hline PEX & 7 & 41.2 & 4 & 23.5 & 2 & 11.8 & 1 & 5.9 & 3 & 17.6 & 17 \\
\hline Secondary & 7 & 22.6 & 2 & 6.5 & 8 & 25.8 & 2 & 6.5 & 12 & 38.7 & 31 \\
\hline Unclassified & 31 & 43.1 & 9 & 12.5 & 10 & 13.9 & 2 & 2.8 & 20 & 27.8 & 72 \\
\hline Total & 683 & 52.7 & 211 & 16.3 & 173 & 13.3 & 35 & 2.7 & 195 & 15.0 & 1297 \\
\hline \multicolumn{12}{|l|}{ a VI visual impairment. } \\
\hline \multicolumn{12}{|c|}{ b $P O A G$ primary open angle glaucoma. } \\
\hline \multicolumn{12}{|l|}{${ }^{\mathrm{c}} O H T$ ocular hypertension. } \\
\hline \multicolumn{12}{|l|}{${ }^{\mathrm{d}} N T G$ normal tension glaucoma. } \\
\hline \multicolumn{12}{|c|}{${ }^{\mathrm{e}} N A G$ narrow angle glaucoma. } \\
\hline${ }^{\mathrm{f}} P D S$ pigment dispersion syn & me. & & & & & & & & & & \\
\hline
\end{tabular}

a severe VI. Other studies reported the prevalence of blindness at the end of life as well, showing different prevalence's, but still a considerable amount of blind deaths $(24.1 \%$ unilateral blindness and $10.6 \%$ bilateral blindness) [15-21]. Differences in operational definition (whether or not including the VF in the definition, which is mostly not counted for in the several definitions that currently exists), the time period in which patients died and regional differences in the prevalence of eye diseases and treatment could explain the differences in prevalence of blindness between these studies.
The findings from our study and other studies have considerable consequences for an aging population. The prevalence of blindness at the end of life will increase, as has been illustrated by studies that predicted the prevalence of glaucoma, blindness and VI [5-7, 22].

These findings also have an important impact for patients since it severely affects their quality of life, especially since low VA contributes to central vision loss in patients who already have lost peripheral VF. As an example, the results showed that $30.5 \%$ of the patients were not allowed to drive solely based on their low VA, 
Table 4 Ranking of most important contributing ocular morbidity to the occurrence of the visual acuity at the end of life with a prevalence of at least $1 \%$, in a cohort of patients with ocular hypertension, glaucoma suspect, or glaucoma.

\begin{tabular}{|c|c|c|c|c|c|c|c|c|}
\hline & Mild $\mathrm{VI}^{\mathrm{a}}$ & $\%$ & Moderate VI & $\%$ & Severe VI & $\%$ & Blind & $\%$ \\
\hline \multirow[t]{18}{*}{ OD } & Cataract & 36.6 & cataract & 23.3 & Glaucoma & 22.2 & Glaucoma & 23.4 \\
\hline & Vision loss e.c.i. ${ }^{b}$ & 21.0 & Dry $\mathrm{AMD}^{\mathrm{c}}$ & 18.0 & Dry $A D^{c}$ & 16.7 & $\mathrm{CRVO}^{\mathrm{d}}$ & 16.2 \\
\hline & Dry $\mathrm{AMD}^{\mathrm{c}}$ & 14.7 & Glaucoma & 16.9 & $\mathrm{CRVO}^{\mathrm{d}}$ & 13.9 & Dry $\mathrm{AMD}^{\mathrm{c}}$ & 7.6 \\
\hline & Glaucoma & 8.9 & Exudative AMD $^{c}$ & 5.2 & Exudative $\mathrm{AMD}^{\mathrm{c}}$ & 8.3 & Past $\mathrm{RD}^{\mathrm{e}}$ & 7.1 \\
\hline & $\mathrm{PCO}^{\mathrm{f}}$ & 3.6 & Vision loss e.c.i. ${ }^{b}$ & 4.7 & Trauma & 5.6 & Exudative $\mathrm{AMD}^{\mathrm{c}}$ & 6.6 \\
\hline & $\mathrm{DRP}^{\mathrm{g}}$ & 2.7 & Amblyopia & 3.5 & Amblyopia & 5.6 & Amblyopia & 6.1 \\
\hline & (Past) keratitis & 2.2 & Corneal dystrophy & 2.9 & $\mathrm{BRVO}^{\mathrm{h}}$ & 5.6 & $\mathrm{DRP}^{\mathrm{g}}$ & 3.0 \\
\hline & Vision assessment without correction & 1.8 & $\mathrm{BRVO}^{\mathrm{h}}$ & 2.3 & Past acute glaucoma & 2.8 & Trauma & 2.0 \\
\hline & Macular pucker & 1.3 & Trauma & 1.7 & $\mathrm{PCO}^{\mathrm{f}}$ & 2.8 & Decompensated cornea & 2.0 \\
\hline & BRVO $^{\mathrm{h}}$ & 1.3 & Past $\mathrm{RD}^{\mathrm{e}}$ & 1.7 & $\mathrm{CRAO}^{\mathrm{i}}$ & 2.8 & $\mathrm{BRVO}^{\mathrm{h}}$ & 2.0 \\
\hline & & & Corneal scar & 1.7 & $\mathrm{AION}^{\mathrm{d}}$ & 2.8 & $\mathrm{RVO}^{\mathrm{j}}$ unspecified & 2.0 \\
\hline & & & $\mathrm{DRP}^{\mathrm{g}}$ & 1.7 & $\mathrm{DRP}^{\mathrm{g}}$ & 2.8 & Enucleation/evisceration/ exenteration & 2.0 \\
\hline & & & Vision assessment without correction & 1.7 & Past $\mathrm{PCR}^{\mathrm{k}}$ and TPPV & 2.8 & Cataract & 1.5 \\
\hline & & & Decompensated cornea & 1.2 & Myopic degeneration & 2.8 & $\mathrm{CRAO}^{\mathrm{i}}$ & 1.5 \\
\hline & & & Past corneal transplantation & 1.2 & Secondary glaucoma & 2.8 & Past keratitis & 1.5 \\
\hline & & & $\mathrm{CRVO}^{\mathrm{d}}$ & 1.2 & & & Vision loss e.c.i. ${ }^{\mathrm{b}}$ & 1.0 \\
\hline & & & BRAO $^{1}$ & 1.2 & & & Corneal transplantation & 1.0 \\
\hline & & & $\mathrm{AION}^{\mathrm{m}}$ & 1.2 & & & $\mathrm{HRVO}^{\mathrm{n}}$ & 1.0 \\
\hline \multirow[t]{18}{*}{ OS } & Cataract & 39.1 & Cataract & 25.3 & Glaucoma & 25.6 & Glaucoma & 24.2 \\
\hline & Vision loss e.c.i. ${ }^{b}$ & 19.1 & Glaucoma & 15.6 & Dry $\mathrm{AMD}^{\mathrm{c}}$ & 25.6 & Dry $A_{M}{ }^{c}$ & 10.9 \\
\hline & Dry $\mathrm{AMD}^{\mathrm{c}}$ & 15.0 & Dry AMD $^{\mathrm{c}}$ & 11.8 & $\mathrm{CRVO}^{\mathrm{d}}$ & 7.7 & $\mathrm{CRVO}^{\mathrm{d}}$ & 9.5 \\
\hline & Glaucoma & 11.4 & Vision loss e.c.i. ${ }^{\mathrm{b}}$ & 10.2 & Vision loss e.c.i. ${ }^{\mathrm{b}}$ & 5.1 & Past $\mathrm{RD}^{\mathrm{e}}$ & 7.6 \\
\hline & $\mathrm{PCO}^{\mathrm{f}}$ & 1.8 & $\mathrm{BRVO}^{\mathrm{h}}$ & 4.8 & Amblyopia & 5.1 & Exudative $\mathrm{AMD}^{\mathrm{c}}$ & 7.1 \\
\hline & Exudative $\mathrm{AMD}^{\mathrm{c}}$ & 1.8 & $\mathrm{DRP}^{\mathrm{g}}$ & 4.8 & Exudative $\mathrm{AMD}^{\mathrm{c}}$ & 5.1 & Enucleation/evisceration/exenteration & 5.2 \\
\hline & Corneal dystrophy & 1.4 & Vision assessment without correction & 3.8 & $\mathrm{HRVO}^{\mathrm{n}}$ & 5.1 & Amblyopia & 4.7 \\
\hline & (past) keratitis & 1.4 & Amblyopia & 2.7 & $\mathrm{RVO}^{\mathrm{j}}$ unspecified & 5.1 & $\mathrm{BRVO}^{\mathrm{h}}$ & 3.8 \\
\hline & $\mathrm{DRP}^{\mathrm{g}}$ & 1.4 & $\mathrm{PCO}^{\mathrm{f}}$ & 2.2 & (past) keratitis & 5.1 & Acute glaucoma & 3.3 \\
\hline & & & Exudative $\mathrm{AMD}^{\mathrm{c}}$ & 2.2 & Corneal transplantation & 2.6 & $\mathrm{AION}^{\mathrm{m}}$ & 2.8 \\
\hline & & & Acute glaucoma & 1.6 & Macular hole & 2.6 & Cataract & 1.9 \\
\hline & & & Trauma & 1.1 & $\mathrm{BRVO}^{\mathrm{h}}$ & 2.6 & $\mathrm{HRVO}^{\mathrm{n}}$ & 1.4 \\
\hline & & & Corneal dystrophy & 1.1 & Secondary glaucoma & 2.6 & $\mathrm{RVO}^{\mathrm{j}}$ unspecified & 1.4 \\
\hline & & & Decompensated cornea & 1.1 & & & $\mathrm{DRP}^{\mathrm{g}}$ & 1.4 \\
\hline & & & Past $\mathrm{RD}^{\mathrm{e}}$ & 1.1 & & & (Past) uveitis & 1.4 \\
\hline & & & Corneal scar & 1.1 & & & Complicated $\mathrm{CE}^{\mathrm{o}}$ & 1.4 \\
\hline & & & (Past) uveitis & 1.1 & & & & \\
\hline & & & Secondary glaucoma & 1.1 & & & & \\
\hline
\end{tabular}

${ }^{\mathrm{a}} V I$ visual impairment.

${ }^{\mathrm{b}}$ eci e causa ignota.

${ }^{\mathrm{c}} A M D$ age-related macular degeneration.

${ }^{\mathrm{d}} C R V O$ central retinal vein occlusion.

${ }^{\mathrm{e}} R D$ retinal detachment.

${ }^{\mathrm{f}} P C O$ posterior capsular opacification.

${ }^{\mathrm{g}} D R P$ diabetic retinopathy.

${ }^{\mathrm{h}} B R V O$ branch retinal vein occlusion.

${ }^{\mathrm{i}} C R A O$ central retinal artery occlusion.

${ }^{\mathrm{j}} R V O$ retinal vein occlusion.

${ }^{\mathrm{k}} P C R$ posterior capsular rupture.

${ }^{\mathrm{l}} B R A O$ branch retinal artery occlusion.

${ }^{\mathrm{m}} A I O N$ anterior ischemic optic neuropathy.

${ }^{\mathrm{n}} H R V O$ hemiretinal vein occlusion.

${ }^{\circ} \mathrm{CE}$ cataract extraction.

according to the requirements of the Dutch agency for driving (CBR). Losing the driving license is known to have a major effect on the quality of life and society as patients lose their independence and mobility [22-26].
Since we investigated glaucoma patients in this study, glaucoma itself contributed to the occurrence of VI and blindness in a considerable number of the patients. As expected, patients with primary open angle glaucoma 
Table 5 Top three most contributing factors for every category of VA per subgroup for OD.

\begin{tabular}{|c|c|c|c|c|c|c|c|c|}
\hline Subgroups & Mild $\mathrm{VI}^{\mathrm{a}}$ & $\%$ & Moderate VI & $\%$ & Severe VI & $\%$ & Blind & $\%$ \\
\hline \multirow[t]{3}{*}{ All } & Cataract & 36.6 & Cataract & 23.3 & Glaucoma & 22.2 & Glaucoma & 23.4 \\
\hline & Vision loss e.c. $\mathrm{i}^{\mathrm{b}}$ & 21.0 & Dry $\mathrm{AMD}^{\mathrm{c}}$ & 18.0 & Dry $\mathrm{AMD}^{\mathrm{c}}$ & 16.7 & $\mathrm{CRVO}^{\mathrm{d}}$ & 16.2 \\
\hline & Dry $\mathrm{AMD}^{\mathrm{c}}$ & 14.7 & Glaucoma & 16.9 & $\mathrm{CRVO}^{\mathrm{d}}$ & 13.9 & Dry $\mathrm{AMD}^{\mathrm{C}}$ & 7.6 \\
\hline \multirow[t]{3}{*}{$\mathrm{POAG}^{\mathrm{e}}$} & Cataract & 37.3 & Cataract & 25.2 & Glaucoma & 33.3 & Glaucoma & 32.5 \\
\hline & Vision loss e.c.i. ${ }^{b}$ & 19.6 & Dry $\mathrm{AMD}^{\mathrm{c}}$ & 21.4 & Dry $\mathrm{AMD}^{\mathrm{c}}$ & 19.0 & $\mathrm{CRVO}^{\mathrm{d}}$ & 15.8 \\
\hline & Dry $\mathrm{AMD}^{\mathrm{c}}$ & 15.0 & Glaucoma & 19.4 & $\mathrm{CRVO}^{\mathrm{d}}$ & 14.3 & Dry $\mathrm{AMD}^{\mathrm{c}}$ & 9.2 \\
\hline \multirow{8}{*}{$\begin{array}{l}\text { POAG }^{\mathrm{d}} \text { suspect, } \\
\text { conversion OHT } \\
\text { to POAG, OHT }\end{array}$} & Cataract & 36.4 & Cataract & 26.7 & Dry $\mathrm{AMD}^{\mathrm{c}}$ & 25.0 & $\mathrm{CRVO}^{\mathrm{d}}$ & 20.0 \\
\hline & Vision loss e.c.i. ${ }^{b}$ & 27.3 & $\mathrm{BRVO}^{\mathrm{g}}$ & 13.3 & Exudative $\mathrm{AMD}^{\mathrm{c}}$ & 25.0 & $\begin{array}{l}\text { Enucleation/ } \\
\text { evisceration/ }\end{array}$ & 20.0 \\
\hline & Dry $\mathrm{AMD}^{\mathrm{c}}$ & 18.2 & Cornea scar & 13.3 & $\mathrm{CRVO}^{\mathrm{d}}$ & 25.0 & exenteration & 10.0 \\
\hline & & & & & $\mathrm{CRAO}^{\mathrm{h}}$ & 25.0 & Dry $\mathrm{AMD}^{\mathrm{c}}$ & 10.0 \\
\hline & & & & & & & Exudative $\mathrm{AMD}^{\mathrm{c}}$ & 10.0 \\
\hline & & & & & & & $\mathrm{BRVO}^{\mathrm{g}}$ & 10.0 \\
\hline & & & & & & & $\mathrm{AION}^{\mathrm{g}}$ & 10.0 \\
\hline & & & & & & & $\begin{array}{l}\text { Past } \mathrm{RD}^{\mathrm{i}} \\
\text { (Past) keratitis }\end{array}$ & 10.0 \\
\hline \multirow[t]{4}{*}{$\mathrm{NAG}^{\mathrm{j}}$} & Cataract & 50.0 & Glaucoma & 28.6 & $\begin{array}{l}\text { None in this } \\
\text { category }\end{array}$ & - & Glaucoma & 42.9 \\
\hline & Dry $\mathrm{AMD}^{\mathrm{c}}$ & 33.3 & Cataract & 28.6 & & & Dry $\mathrm{AMD}^{\mathrm{c}}$ & 28.6 \\
\hline & Vision loss e.c.i. ${ }^{b}$ & 16.7 & Dry $\mathrm{AMD}^{\mathrm{c}}$ & 21.4 & & & Amblyopia & 14.3 \\
\hline & & & & & & & $\begin{array}{l}\text { Bleeding in the } \\
\text { past e.c.i. }\end{array}$ & 14.3 \\
\hline \multirow[t]{7}{*}{$\mathrm{NTG}^{\mathrm{k}}$} & Cataract & 50.0 & Glaucoma & 25.0 & Acute glaucoma & 50.0 & $\mathrm{CRVO}^{\mathrm{d}}$ & 50.0 \\
\hline & Glaucoma & 16.7 & Vision loss e.c.i. ${ }^{\text {b }}$ & 12.5 & Exudative $\mathrm{AMD}^{\mathrm{c}}$ & 50.0 & Cataract & 25.0 \\
\hline & Dry AMD ${ }^{c}$ & 16.7 & Amblyopia & 12.5 & & & Ischemia & 25.0 \\
\hline & & & Acute glaucoma & 12.5 & & & & \\
\hline & & & Cataract & 12.5 & & & & \\
\hline & & & Dry $\mathrm{AMD}^{\mathrm{c}}$ & 12.5 & & & & \\
\hline & & & $\mathrm{DRP}^{1}$ & 12.5 & & & & \\
\hline \multirow[t]{6}{*}{ Others } & 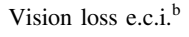 & 26.3 & Amblyopia & 10.5 & Trauma & 16.7 & $\mathrm{CRVO}^{\mathrm{d}}$ & 17.8 \\
\hline & & 21.1 & Glaucoma & 10.5 & Glaucoma & 16.7 & Past $\mathrm{RD}^{\mathrm{i}}$ & 13.3 \\
\hline & Cataract & 10.5 & Cataract & 10.5 & $\mathrm{AION}^{\mathrm{m}}$ & 16.7 & Glaucoma & 8.9 \\
\hline & $\mathrm{DRP}^{1}$ & & Dry $\mathrm{AMD}^{\mathrm{c}}$ & 10.5 & $\mathrm{DRP}^{\mathrm{l}}$ & 16.7 & & \\
\hline & & & Exudative AMD $^{\mathrm{c}}$ & 10.5 & $\begin{array}{l}\text { Myopic } \\
\text { degeneration }\end{array}$ & 16.7 & & \\
\hline & & & & & $\begin{array}{l}\text { Secondary } \\
\text { glaucoma }\end{array}$ & 16.7 & & \\
\hline
\end{tabular}

${ }^{\mathrm{a}} V I$ visual impairment.

${ }^{\mathrm{b}}$ eci e causa ignota.

${ }^{\mathrm{c}} A M D$ age-related macular degeneration.

${ }^{\mathrm{d}} C R V O$ central retinal vein occlusion.

${ }^{\text {e }} P O A G$ primary open angle glaucoma.

${ }^{\mathrm{f}} \mathrm{OHT}$ ocular hypertension.

${ }^{\mathrm{g}} B R V O$ branch retinal vein occlusion.

${ }^{\mathrm{h}} C R A O$ central retinal artery occlusion.

${ }^{\mathrm{i}} R D$ retinal detachment.

${ }^{\mathrm{j}} N A G$ narrow angle glaucoma.

${ }^{\mathrm{k}} N T G$ normal tension glaucoma

${ }^{1} D R P$ diabetic retinopathy.

${ }^{\mathrm{m}} A I O N$ anterior ischemic optic neuropathy.

are more at risk than glaucoma suspect or ocular hypertension patients. There is no apparent difference in risk between patients with primary open angle glaucoma, normal tension glaucoma or narrow angle glaucoma, however if VF was included there would probably be a substantial difference in risk between these diseases. Table 6 indeed shows already that glaucoma is the highest ranking contributing factor in POAG as compared with OHT/glaucoma suspect/conversion as expected, the risk is higher in patients with secondary glaucoma, most likely 
due to the underlying cause of the glaucoma and its complications.

The observation that glaucoma is a relevant contributing factor could be either due to an advanced stage of glaucoma at diagnosis or a progressive course, or both [18, 27]. Patient delays and delays in the health care system may contribute to a late diagnosis [28]. Screening and case finding is an option to improve the time to diagnosis. It has been shown that case finding by the ophthalmologist by measuring the IOP when a patient comes for other eye complaints is a cost-effective approach [29]. If a more progressive course has contributed to the occurrence of blindness, more aggressive lowering IOP while monitoring the disease could have prevented this. Moreover, a strategy to decrease IOP from the start of diagnosis to a lower target instead of lowering the target-pressure step by step when progression has occurred, prevents more blind deaths [30]. In addition to preventing VI and blindness due to glaucoma itself this strategy could possibly also prevent RVO which occurs more often in glaucoma patients $[11,12]$.

The main contributing eye diseases to the occurrence of severe VI or blindness according to the VA at the end of life, besides glaucoma itself, were among others RVO, AMD, retinal detachment, amblyopia, DRP, and cataract. All these factors can potentially be prevented or treated. Prevention is by means of early amblyopia discovery and treatment, primary prevention by means of lifestyle changes or medical treatment of cardiovascular risk factors and diabetes and preventing patient and GP delay in case of complaints of retinal detachment. Moreover, the ophthalmologist could prevent the occurrence of ocular comorbidity in the eye for some of the contributing factors.

Treatment by the ophthalmologist is also a possible option. The presence of cataract as a prevalent contributing factor to mild and moderate VI suggests that treatment of either comorbidity would be beneficial. Treatment of exudative AMD, DRP, RVO, and retinal detachment are also within the realm of the ophthalmologist's possibilities. Whether improvements in the quality of care could have contributed to the prevention of a blind death or improvement in VI needs to be studied. In any case, glaucoma treatment is more than lowering the IOP.

Despite the interesting and important findings of this study, there are some issues that should be discussed.

The strengths of the current study are random selection of hospitals, the long follow-up and the data represent what one observes in daily practice, which gives a more representative picture of the patient. Moreover, the inclusion of a large cohort of patients from the DURING study that represents a large catchment area. This gives a higher chance of having data that are representative for the catchment area, e.g., the Netherlands. The DURING study included patients from nine randomly selected hospitals of which teaching, university and general hospitals and from different parts of the Netherlands which included the north, middle and south parts of the Netherlands.

One of the limitations of the current study was missing data, which can be expected in performing a retrospective study. Sixteen percent of the patients had to be excluded due to incomplete data or missing patient files. Some hospitals at some point made the transition from paper patient files to electronic files, some hospitals relocated and others merged; these changes mostly explain why patient files or data were missing. Another difficulty was that some files were not clearly written or had missing diagnoses.

Data of some patients were missing or not complete due to loss to follow-up, probably due to moving of the patient or referral to another (university) hospital for further treatment. The latter could be more problematic since this is related to the severity and/or progression of glaucoma. The comparison between hospital types did show a small difference in the prevalence of VI or blindness at the end of life. However, the university hospital Maastricht is the only hospital in the vicinity of Maastricht and also has a regional function and one of the other hospitals was a large topclinical hospital where also invasive glaucoma surgery was conducted, limiting the number of referrals.

Furthermore, the study was based on patient record forms and therefore diagnoses and assessment of VA and their contributing factors could not be based on uniform and rigorous methods to assess these.

The mean time between last measured VA and death was 2 years. This could be due to referral of the patient, moving of the patient or nonattendance for follow-up for example because the patient was too ill at the end of life or had an untreatable eye condition. This may underestimate risk of blindness. The follow-up is not complete since not all, although a considerable number, of the included patients have died. One could question if this has affected the ranking of contributing factors or prevalence of blindness. For example, if a patient suffered from a CRVO, which is known to be related to cardiovascular risk factors, this patient is more likely to die sooner as compared with patients with some other contributing factors.

Furthermore, we did not perform an epidemiological study in which all participants have the same examinations and statistical analysis can be conducted to assess relations. Therefore, we took a clinical approach in deciding on whether a factor was a contributing factor. In clinical practice we commonly have to make our decision on the cause of vision loss on the basis of the medical history and examination of the eye. We therefore carefully studied the medical file of the ophthalmologist to identify contributing factors.

In addition, in the current study we used the VF only in the assessment of central VA in case no explanation was found for a decline in VA (as described in the "Methods" section), but we did not use the VF in assessing blindness due to VF 
defect since this was not the objective of this study. However, if we did use the VF's in assessing blindness, probably the prevalence of blindness would be much higher since there are patients who have a good VA but a VF which is restricted to 10 degrees in a radius around central fixation for example, which is classified as blind according to the WHO.

The percentage of blindness due to glaucoma is lower in our study compared with percentages in the studies of Peters et al. and Forsman et al. which showed that $15 \%$ of glaucoma patient became blind. This can be explained by the fact that both these studies included the VF in the assessment of blindness and our study only included the VA. However, the goal of our study was to look only at the effect of VA on the blindness prevalence in the glaucoma patients.

Finally, we studied the patients from the cohort of the DURING study which had a high response rate of $79 \%$, but still several patients have not been included. This could be for reasons related to the severity of their eye diseases. These subjects have a higher risk of becoming blind. Our estimates of the prevalence of VI and blindness at the end of life may therefore be too low.

In conclusion, the prevalence of VI and blindness, based on VA, in glaucoma patients at the end of life is high. Glaucoma itself and several other eye diseases for which prevention and treatment is possible contribute to their occurrence. Early diagnosis of glaucoma is warranted and management of glaucoma need to be intensified. Moreover, management of glaucoma patient entails more than lowering the IOP to prevent a blind death, i.e., the treatment of contributing eye diseases such as discussed above, which contribute to the occurrences of blindness also in glaucoma patients and therefore need to be of concern in treating glaucoma patients. And even more than in patients without glaucoma. If for example prevention of AMD by means of nutritional supplements in glaucoma patients is not addressed, the impact in glaucoma patients would be higher. They lose peripheral VF as well as central VA.

\section{Summary}

\section{What was known before}

- As far as we know, no study has investigated the level of visual acuity at the end of life in glaucoma and its contributing factors in the extent we do.

\section{What this study adds}

- Our study shows that not only glaucoma, but also other eye diseases contribute to end of life blindness in glaucoma patient.
- Some of which can be prevented or treated on time.

Funding The author was supported by the following foundations: algemene Nederlandse vereniging ter voorkoming van blindheid, glaucoom fonds and landelijke stichting voor blinden en slechtzienden that contributed through UitZicht. The funding organizations had no role in the design or conduct of this research.

\section{Compliance with ethical standards}

Conflict of interest The authors declare that they have no conflict of interest.

Publisher's note Springer Nature remains neutral with regard to jurisdictional claims in published maps and institutional affiliations.

\section{References}

1. Cook C, Foster P. Epidemiology of glaucoma: what's new? Can J Ophthalmol. 2012;47:223-6.

2. Mantravadi AV, Vadhar N. Glaucoma. Prim Care. 2015;42:437-49.

3. Gupta D, Chen PP. Glaucoma. Am Fam Physician. 2016;93:668-74.

4. King A, Azuara-Blanco A, Tuulonen A. Glaucoma. BMJ. 2013;346: f3518.

5. Flaxman SR, Bourne RRA, Resnikoff S, Ackland P, Braithwaite $\mathrm{T}$, Cicinelli MV, et al. Global causes of blindness and distance vision impairment 1990-2020: a systematic review and metaanalysis. Lancet Glob Health. 2017;5:e1221-e34.

6. Bourne RR, Taylor HR, Flaxman SR, Keefe J, Leasher J, Naidoo $\mathrm{K}$, et al. Number of people blind or visually impaired by glaucoma worldwide and in World Regions 1990-2010: a meta-analysis. PLoS One. 2016;11:e0162229.

7. Tham YC, Li X, Wong TY, Wong TY, Quigley HA, Aung T, et al. Global prevalence of glaucoma and projections of glaucoma burden through 2040: a systematic review and meta-analysis. Ophthalmology. 2014;121:2081-90.

8. Pelletier AL, Rojas-Roldan L, Coffin J. Vision loss in older adults. Am Fam Physician. 2016;94:219-26.

9. Zetterberg M. Age-related eye disease and gender. Maturitas. 2016;83:19-26.

10. Rehak M, Wiedemann P. Retinal vein thrombosis: pathogenesis and management. J Thromb Haemost. 2010;8:1886-94.

11. Kolar P. Risk factors for central and branch retinal vein occlusion: a meta-analysis of published clinical data. J Ophthalmol. 2014; 2014:724780.

12. Yin X, Li J, Zhang B, Lu P. Association of glaucoma with risk of retinal vein occlusion: a meta-analysis. Acta Ophthalmol. 2019; 97:652-9.

13. van der Valk R, Webers CA, Hendrikse F, de Vogel SC, Prins MH, Schouten JSAG. et al. Predicting intraocular pressure change before initiating therapy: timolol versus latanoprost. Acta Ophthalmol. 2008;86:415-8.

14. Schulze-Bonsel K, Feltgen N, Burau H, Hansen L, Bach M. Visual acuities "hand motion" and "counting fingers" can be quantified with the freiburg visual acuity test. Investig Ophthalmol Vis Sci. 2006;47:1236-40.

15. Peters D, Bengtsson B, Heijl A. Lifetime risk of blindness in open-angle glaucoma. Am J Ophthalmol. 2013;156:724-30.

16. Ang GS, Eke T. Lifetime visual prognosis for patients with primary open-angle glaucoma. Eye. 2007;21:604-8.

17. Mokhles P, Schouten JS, Beckers HJ, Azuara-Blanco A, Tuulonen A, Webers CAB. A systematic review of end-of-life 
visual impairment in open-angle glaucoma: an epidemiological autopsy. J Glaucoma. 2016;25:623-8.

18. Mokhles P, Schouten JS, Beckers HJ, Azuara-Blanco A, Tuulonen A, Webers CAB. Glaucoma blindness at the end of life. Acta Ophthalmol. 2017;95:10-1.

19. Ernest PJ, Busch MJ, Webers CA, Beckers HJM, Hendrikse F, Prins $\mathrm{MH}$, et al. Prevalence of end-of-life visual impairment in patients followed for glaucoma. Acta Ophthalmol. 2013;91:738-43.

20. Forsman E, Kivela T, Vesti E. Lifetime visual disability in openangle glaucoma and ocular hypertension. J Glaucoma. 2007;16: 313-9.

21. Goh YW, Ang GS, Azuara-Blanco A. Lifetime visual prognosis of patients with glaucoma. Clin Exp Ophthalmol. 2011;39:766-70.

22. Hochberg C, Maul E, Chan ES, van Landingham S, Ferrucci L, Friedman DS, et al. Association of vision loss in glaucoma and age-related macular degeneration with IADL disability. Investig Ophthalmol Vis Sci. 2012;53:3201-6.

23. Blane A. Through the looking glass: a review of the literature investigating the impact of glaucoma on crash risk, driving performance, and driver self-regulation in older drivers. J Glaucoma. 2016;25:113-21.
24. Montana CL, Bhorade AM. Glaucoma and quality of life: fall and driving risk. Curr Opin Ophthalmol. 2018;29:135-40.

25. Sachdev A, Tahhan M, Sung VCT. Glaucoma and driving: are we documenting driving status and advising patients with glaucoma appropriately about their driving? Int Ophthalmol. 2018;38:419-23.

26. Gramer G, Gramer E. Stage of visual field loss and age at diagnosis in 1988 patients with different glaucomas: implications for glaucoma screening and driving ability. Int Ophthalmol. 2018;38:429-41.

27. Peters D, Bengtsson B, Heijl A. Factors associated with lifetime risk of open-angle glaucoma blindness. Acta Ophthalmol. 2014;92:421-5.

28. Prior M, Francis JJ, Azuara-Blanco A, Anand N, Burr JM. Why do people present late with advanced glaucoma? A qualitative interview study. Br J Ophthalmol. 2013;97:1574-8.

29. Peeters A, Schouten JS, Webers CA, Prins MH, Hendrikse F, Severens JL. Cost-effectiveness of early detection and treatment of ocular hypertension and primary open-angle glaucoma by the ophthalmologist. Eye. 2008;22:354-62.

30. van Gestel A, Webers CA, Severens JL, Beckers HJ, Jansonius NM, Hendrikse F, et al. The long-term outcomes of four alternative treatment strategies for primary open-angle glaucoma. Acta Ophthalmol. 2012;90:20-31. 